\title{
Ad Hoc Committee Shares Early Results of Professional Development Initiative
}

\section{Lynne Robinson}

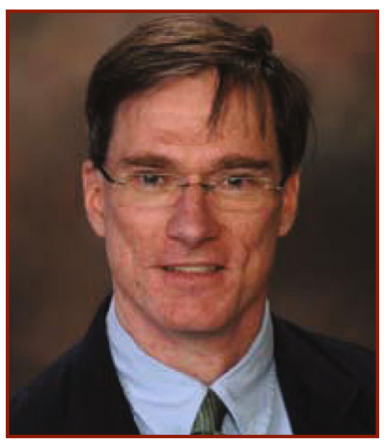

Jeffrey Fergus

"Professional

development needs are

different for different people and in different stages of their careers."

一Jeffrey Fergus, Ad Hoc Professional Development Committee Chair
For additional
information on
professional
development at
TMS, and to propose
a new professional
development
event, visit

www.tms.org/pd or

contact the

TMS Professional

Development staff

at pd@tms.org.
In just a few months, the TMS Ad Hoc Professional Development Committee has already made an impact on what, how, when, and where TMS members can access resources and opportunities to help them advance in their careers.

Formally established by the TMS Board of Directors in October 2014, the committee is responsible for evaluating and coordinating TMS activities for both technical and nontechnical professional development programs. In developing its strategies, the committee has focused on tailoring content and training approaches to specific member groups within TMS. "Professional development needs are different for different people and in different stages of their careers," explained Jeffrey Fergus, Committee Chair and TMS Board Director, Professional Development. "Young professionals may need advice and mentorship to advance as they begin their careers. Later, individuals may move into different roles or new technical areas and could benefit from support during these transitions."

Among the first new events developed by the committee along these lines is Meet a Mentor, scheduled for the TMS 2015 Annual Meeting and Exhibition (TMS2015) on March 16. Using the same techniques as speed networking, offered at TMS2014, Meet a Mentor pairs those who would like to move into a mentoring role with younger professionals who are seeking mentors.

Also at TMS2015, the traditional slate of technical workshops and short courses offered on March 15 has been enriched with several new types of professional development opportunities. These include a workshop geared to young scientists on professional skills necessary to ensure career success.

Throughout 2015 and beyond, TMS is developing multi-day courses focused on the development of specific technical skill sets. These include the Aluminum Cast Shop Science and Technology Course, May 10-15 in Evansville, Indiana, and a short course, Implementing ICME in Industry, to be held June 4-5 in conjunction with the 3rd World Congress on Integrated Computational Materials Engineering. Plans are also underway to offer a preparation course in August for the October 2015 Metallurgical and Materials Professional Engineering Examination.

In addition, TMS is exploring how webinar technologies can be effectively used to deliver timely professional development content to its members at their convenience. The Impact of Contextualized Computing for Materials Students-TMS's first webinar in this effort—was broadcast live in November 2014 and offered valuable insights into how this model of professional development training can continue to be enhanced.

While technical topics will remain a cornerstone for TMS's professional development efforts, the ad hoc committee is placing a particular emphasis on cultivating opportunities that address nontechnical training needs and is encouraging proposals for new professional development events. Details on program formats and approaches, as well as a program proposal form, are available at www.tms.org/pd/events/proposal.

While TMS has offered professional development programming in the past, Fergus believes that these efforts are now poised to evolve to a new level of value and service to TMS members.

"The creation of the ad hoc committee signals a commitment to provide support to TMS members," he said. "I am looking forward to seeing how this initiative will lead to the development of programs and services that will help support TMS members individually to advance along their career paths."

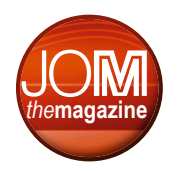

